\title{
Long lasting spasticity in controlled vasospastic angina
}

\author{
O Ueda, K Kohchi, Y Kishi, F Numano
}

Department of Cardiology, Chiba

Tokushukai Hospital, 1-27-1 Narashinodai, Funabashishi, Chiba 274 8503, Japan

O Ueda

K Kohchi

The Third Department of Internal Medicine, Tokyo Medical and Dental University, Tokyo, Japan

Y Kishi

F Numano

Correspondence to: Dr Ueda.

Accepted for publication 4 December 1998

\begin{abstract}
Objective-To evaluate changes in coronary artery spasticity in patients with vasospastic angina who had been stable for years under continuous drug treatment.

Methods-Follow up coronary angiography was performed under intracoronary ergonovine provocation in 27 well controlled patients with vasospastic angina and no organic stenosis; the tests were done $>24$ months after the initial coronary angiography, in which occlusive spasm had been induced by the same regimen of ergonovine provocation.

Results-The mean (SD) follow up period was 47.2 (21.6) months. All patients had been free from angina attack for more than 24 months under treatment with antianginal drugs. During this follow up period, organic stenosis developed in only one case. Occlusive spasm was observed during follow up coronary angiography in 23 patients. Spasm with $90 \%$ narrowing was observed in three other patients, and diffuse significant narrowing was seen in the final patient. No significant difference was found in spasticity $(p=0.75)$ between the initial and the follow up tests.

Conclusions-Repeated ergonovine provocation during coronary angiography after
\end{abstract}

a controlled period of several years showed that coronary spasm remains inducible in most patients. Discontinuance of drug treatment during the remission from anginal attacks achieved by medication may put the patient at high risk.

(Heart 1999;81:528-532)

Keywords: vasospastic angina; ergonovine; angiography

The significance of coronary arterial spasm in ischaemic heart disease is now widely understood $^{12}$ and the provocation test during coronary angiography has come into general use in the diagnosis. Various studies on the prognosis of vasospastic angina have been reported. ${ }^{3-7}$ Most patients with vasospastic angina who have no significant organic stenosis are easily managed by antianginal agents, including long acting nitrates, and their prognosis appears to be satisfactory. There remains, however, the question of whether to continue drug treatment indefinitely. The risks of withdrawal and the possibility of spontaneous remission have both been pointed out. ${ }^{6}{ }^{8}$ It is important to know whether drugs can safely be discontinued, particularly in cases of pure vasospastic angina without significant coronary artery stenosis.

Table 1 Clinical and angiographic characteristics of the patients

\begin{tabular}{|c|c|c|c|c|c|c|c|c|c|}
\hline \multirow[b]{2}{*}{ No } & \multirow[b]{2}{*}{$\begin{array}{l}\text { Age } \\
\text { (years)/sex }\end{array}$} & \multirow[b]{2}{*}{$\begin{array}{l}\text { Follow up } \\
\text { (months) }\end{array}$} & \multirow[b]{2}{*}{ Drugs during follow up } & \multicolumn{3}{|l|}{ Initial test } & \multicolumn{3}{|l|}{ Follow up test } \\
\hline & & & & $\begin{array}{l}\text { ST segment } \\
\text { changes }\end{array}$ & $\begin{array}{l}\text { Site of spasm } \\
\text { (seg) }\end{array}$ & $\begin{array}{l}\text { Chest } \\
\text { pain }\end{array}$ & $\begin{array}{l}\text { ST segment } \\
\text { changes }\end{array}$ & $\begin{array}{l}\text { Site of spasm } \\
\text { (seg) }\end{array}$ & $\begin{array}{l}\text { Chest } \\
\text { pain }\end{array}$ \\
\hline 1 & $79 \mathrm{M}$ & 83 & sr.ISDN $40 \mathrm{mg}$, ISDN $5 \mathrm{mg}$, sr.nif $20 \mathrm{mg}$, dil $30 \mathrm{mg}$ & $2,3, \mathrm{~F} \uparrow$ & 1 & + & $2,3, \mathrm{~F} \uparrow$ & 2 & + \\
\hline 2 & $77 \mathrm{M}$ & 56 & sr.ISDN $40 \mathrm{mg}$, sr.nif $20 \mathrm{mg}$ & 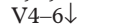 & 11 & + & $\mathrm{V} 2-5 \uparrow$ & 7,11 & + \\
\hline 3 & $63 \mathrm{M}$ & 48 & sr.ISDN $40 \mathrm{mg}$, sr.nif $40 \mathrm{mg}$, nic $20 \mathrm{mg}$ & - & 1 & + & - & 1 & + \\
\hline 4 & $72 \mathrm{M}$ & 36 & sr.ISDN $40 \mathrm{mg}$, dil $60 \mathrm{mg}$ & - & 7 & + & $2,3, \mathrm{~F} \uparrow$ & 3 & + \\
\hline 5 & $68 \mathrm{M}$ & 93 & sr.ISDN $40 \mathrm{mg}$, dil $60 \mathrm{mg}$ & 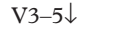 & 6,11 & + & 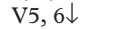 & 11 & + \\
\hline 6 & $84 \mathrm{M}$ & 25 & sr.ISDN $40 \mathrm{mg}$, sr.nif $20 \mathrm{mg}$ & - & 7,11 & + & - & $-(4 ; 90 \%)$ & - \\
\hline 7 & $60 \mathrm{M}$ & 79 & sr.ISDN $40 \mathrm{mg}$, sr.nif $20 \mathrm{mg}$ & - & 1 & + & $2,3, \mathrm{~F} \downarrow$ & 11 & + \\
\hline 8 & $65 \mathrm{M}$ & 25 & sr.ISDN $40 \mathrm{mg}$, sr.dil $200 \mathrm{mg}$ & $\mathrm{V} 2-5 \uparrow$ & 6 & + & $\mathrm{V} 2-6 \uparrow$ & 6,11 & + \\
\hline 9 & $50 \mathrm{M}$ & 50 & sr.ISDN $40 \mathrm{mg}$, sr.nif $20 \mathrm{mg}$, nic $15 \mathrm{mg}$ & - & 1 & + & - & $-(1 ; 90 \%)$ & - \\
\hline 10 & $75 \mathrm{M}$ & 35 & sr.ISDN $40 \mathrm{mg}$, td.NTG $25 \mathrm{mg}$, sr.nif $40 \mathrm{mg}$ & $\mathrm{V} 1-6 \uparrow$ & 7 & - & - & 7 & + \\
\hline 11 & $64 \mathrm{M}$ & 31 & sr.ISDN $80 \mathrm{mg}$, td.ISDN $40 \mathrm{mg}$, sr.nif $80 \mathrm{mg}$, nic $20 \mathrm{mg}$ & $2,3, \mathrm{~F} \uparrow$ & 2 & + & - & 2 & + \\
\hline 12 & $61 \mathrm{M}$ & 52 & sr.ISDN $40 \mathrm{mg}$, sr.nif $20 \mathrm{mg}$ & $\mathrm{V} 1-4 \uparrow$ & 6 & + & $\mathrm{V} 4-6 \uparrow$ & 6,12 & + \\
\hline 13 & $62 \mathrm{M}$ & 35 & sr.ISDN $40 \mathrm{mg}$, benidipine $8 \mathrm{mg}$, nic $15 \mathrm{mg}$ & - & 12 & - & - & 12 & + \\
\hline 14 & $45 \mathrm{M}$ & 24 & td.ISDN $40 \mathrm{mg}$, sr.nif $40 \mathrm{mg}$ & $\mathrm{V} 2-5 \uparrow$ & 6 & + & - & $-(6 ; 90 \%)$ & - \\
\hline 15 & $50 \mathrm{M}$ & 71 & sr.ISDN $40 \mathrm{mg}$, dil $30 \mathrm{mg}$ & - & 11 & + & $2,3, \mathrm{~F} \uparrow$ & 2 & + \\
\hline 16 & $64 \mathrm{M}$ & 49 & sr.ISDN $40 \mathrm{mg}$, sr.nife $20 \mathrm{mg}$, nic $10 \mathrm{mg}$ & $2,3, \mathrm{~F} \uparrow$ & 3 & + & $\mathrm{V} 2-6 \uparrow$ & 7,11 & + \\
\hline 17 & $57 \mathrm{M}$ & 31 & sr.ISDN $40 \mathrm{mg}$, dil $60 \mathrm{mg}$ & - & 9 & - & $2,3, \mathrm{~F}, \mathrm{~V} 6 \uparrow$ & 9,11 & + \\
\hline 18 & $58 \mathrm{M}$ & 94 & sr.ISDN $40 \mathrm{mg}$, sr.nif $10 \mathrm{mg}$, dil $30 \mathrm{mg}$, nic $5 \mathrm{mg}$ & $2,3, \mathrm{~F} \uparrow$ & 1 & + & $\mathrm{V} 1-3 \uparrow$ & 6 & + \\
\hline 19 & $66 \mathrm{M}$ & 67 & sr.ISDN $40 \mathrm{mg}$, sr.nif $20 \mathrm{mg}$ & $\mathrm{V} 1-4 \uparrow$ & 6 & + & $\mathrm{V} 1-3 \uparrow$ & 6 & + \\
\hline 20 & $64 \mathrm{~F}$ & 29 & td.ISDN $40 \mathrm{mg}$ & $\mathrm{V} 3-6 \uparrow$ & 11 & + & $\mathrm{V} 3-6 \uparrow$ & 7,11 & + \\
\hline 21 & $67 \mathrm{M}$ & 49 & sr.ISDN $40 \mathrm{mg}$, sr.nif $20 \mathrm{mg}$ & $2,3, \mathrm{~F} \uparrow$ & 2 & + & $2,3, \mathrm{~F} \uparrow$ & 2 & + \\
\hline 22 & $71 \mathrm{M}$ & 28 & sr.ISDN $40 \mathrm{mg}$, sr.nif $20 \mathrm{mg}$ & $2 \uparrow$ & 13 & + & $2,3, \mathrm{~F} \uparrow$ & 2 & + \\
\hline 23 & $62 \mathrm{M}$ & 53 & sr.ISDN $40 \mathrm{mg}$, sr.nif $20 \mathrm{mg}$ & $\mathrm{V} 5,6 \uparrow$ & 11 & + & - & - & + \\
\hline 24 & $54 \mathrm{~F}$ & 60 & sr.ISDN $40 \mathrm{mg}$, sr.nif $20 \mathrm{mg}$ & $2,3, \mathrm{~F} \uparrow$ & 1 & + & $2,3, \mathrm{~F} \uparrow$ & 2 & + \\
\hline 25 & $64 \mathrm{M}$ & 24 & sr.ISDN $40 \mathrm{mg}$, sr.nif $30 \mathrm{mg}$ & $2,3, \mathrm{~F} \uparrow$ & 1 & + & $\mathrm{V} 3-6 \uparrow$ & 7 & + \\
\hline 26 & $67 \mathrm{M}$ & 24 & sr.ISDN $40 \mathrm{mg}$, dil $60 \mathrm{mg}$ & - & 12 & + & $\mathrm{V} 2-4 \uparrow$ & 7,11 & + \\
\hline 27 & $43 \mathrm{M}$ & 24 & td.ISDN $40 \mathrm{mg}$, dil $60 \mathrm{mg}$, nic $10 \mathrm{mg}$ & $\mathrm{V} 2-6 \uparrow$ & 7 & + & $\mathrm{V} 2-6 \uparrow$ & 7 & + \\
\hline
\end{tabular}

dil, diltiazem; ISDN, isosorbide dinitrate; nic, nicorandil; nif, nifedipine; sr, sustained release; td, transdermal; seg, coronary segment as defined by the American Heart Association classification; $\uparrow$, ST segment elevation; $\downarrow$, ST segment depression. ${ }^{10}$ 
We previously reported that a diagnostic provocation test using one dose of ergonovine into each coronary artery is simpler, easier, and safer than various other provocation methods. ${ }^{9}$ We have been employing this regimen since 1988. In this paper we report the changes in coronary artery spasticity in patients who had been stable for years, and discuss the value of sustained medical treatment.
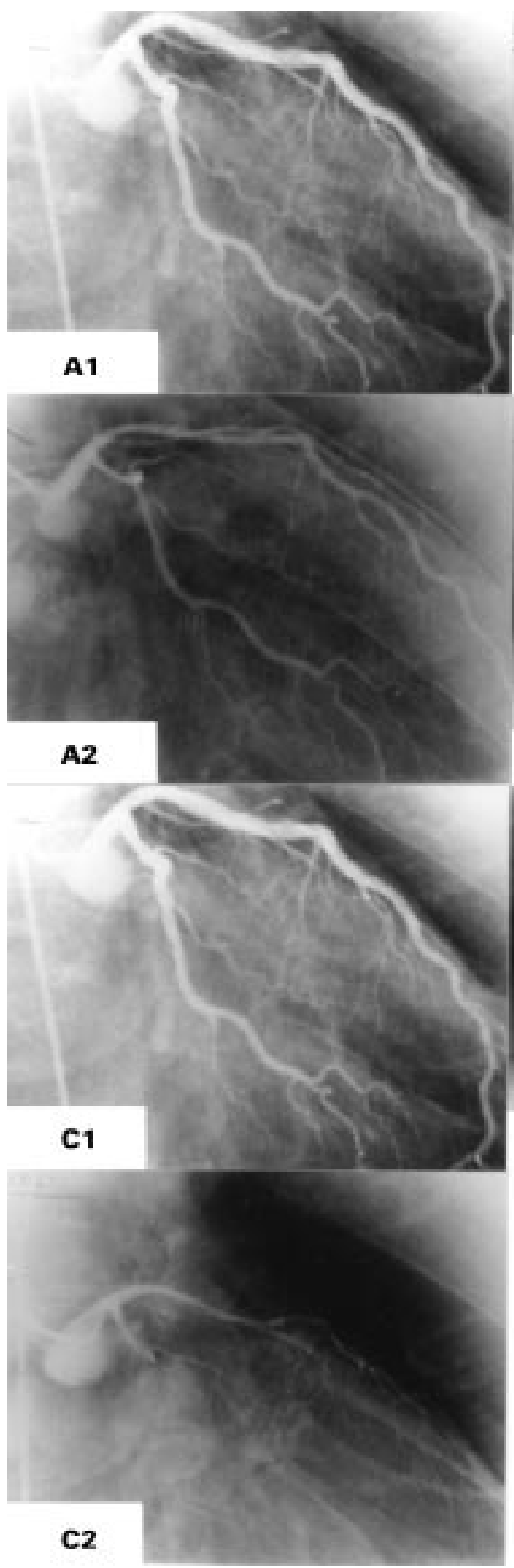

\section{Methods}

PATIENTS

Between November 1988 and March 1995, 107 patients developed occlusive spasm under ergonovine provocation during coronary angiography at the Chiba Tokushukai Hospital. Thirty seven patients without organic stenosis ( $>50 \%$ luminal narrowing) in any coronary artery and with no history of

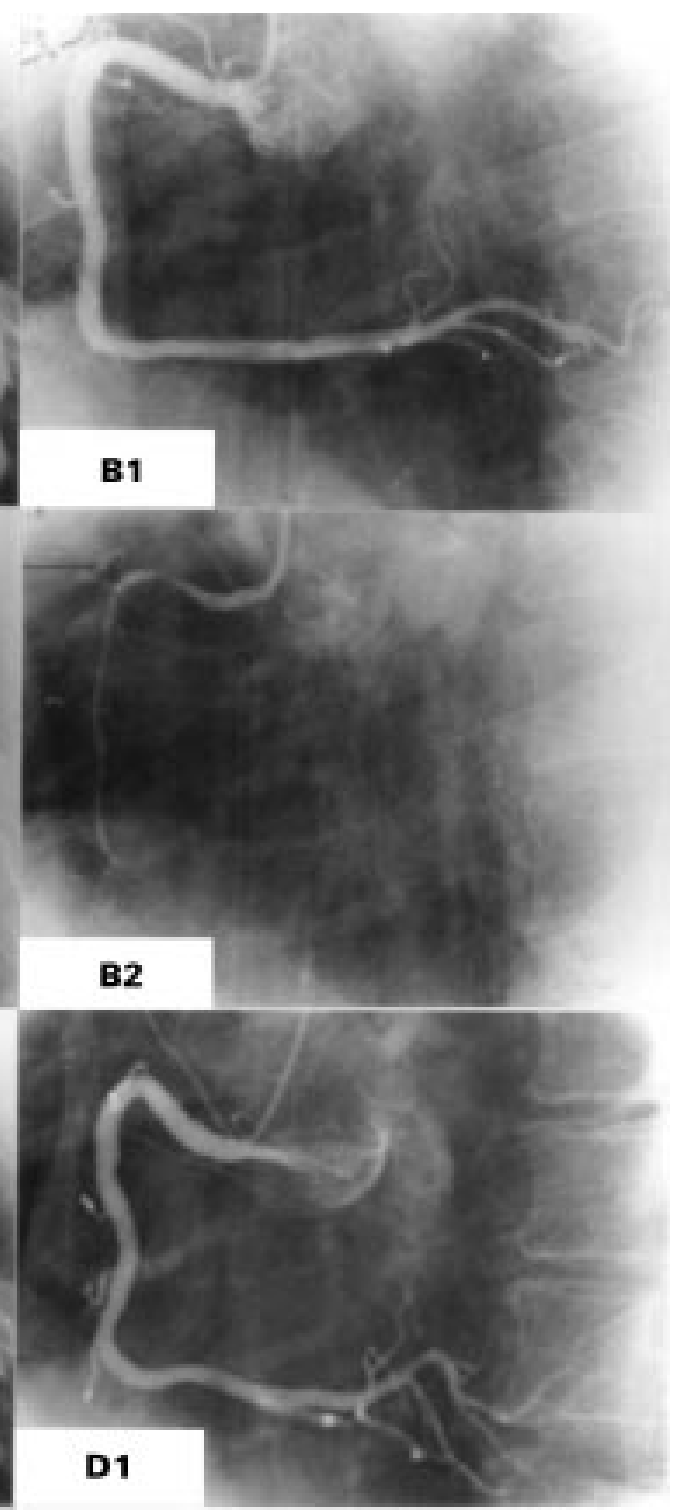

Figure 1 Initial and follow up coronary angiograms from patient 16. Angiograms of $A 1, A 2, C 1$, and $C 2$ of the left coronary artery were obtained in the right anterior oblique projection; angiograms $B 1, B 2$, and D1 of the right coronary artery were obtained in the left anterior oblique projection. Angiograms were obtained after administration of $20 \mu \mathrm{g}$ of intracoronary ergonovine (A2, B2, and C2), or after administration of $5 \mathrm{mg}$ of intracoronary isosorbide dinitrate $(A 1, B 1, C 1$, and $D 1)$. On the initial angiogram, occlusive spasm was observed in American Heart

Association (AHA) segment 3 after the administration of ergonovine (B2), but no significant organic stenosis was present after administration of isosorbide dinitrate ( $A 1$ and B1). On the follow up angiogram, occlusive spasm was observed in AHA segments 7 and 11 after administration of ergonovine (C2). 

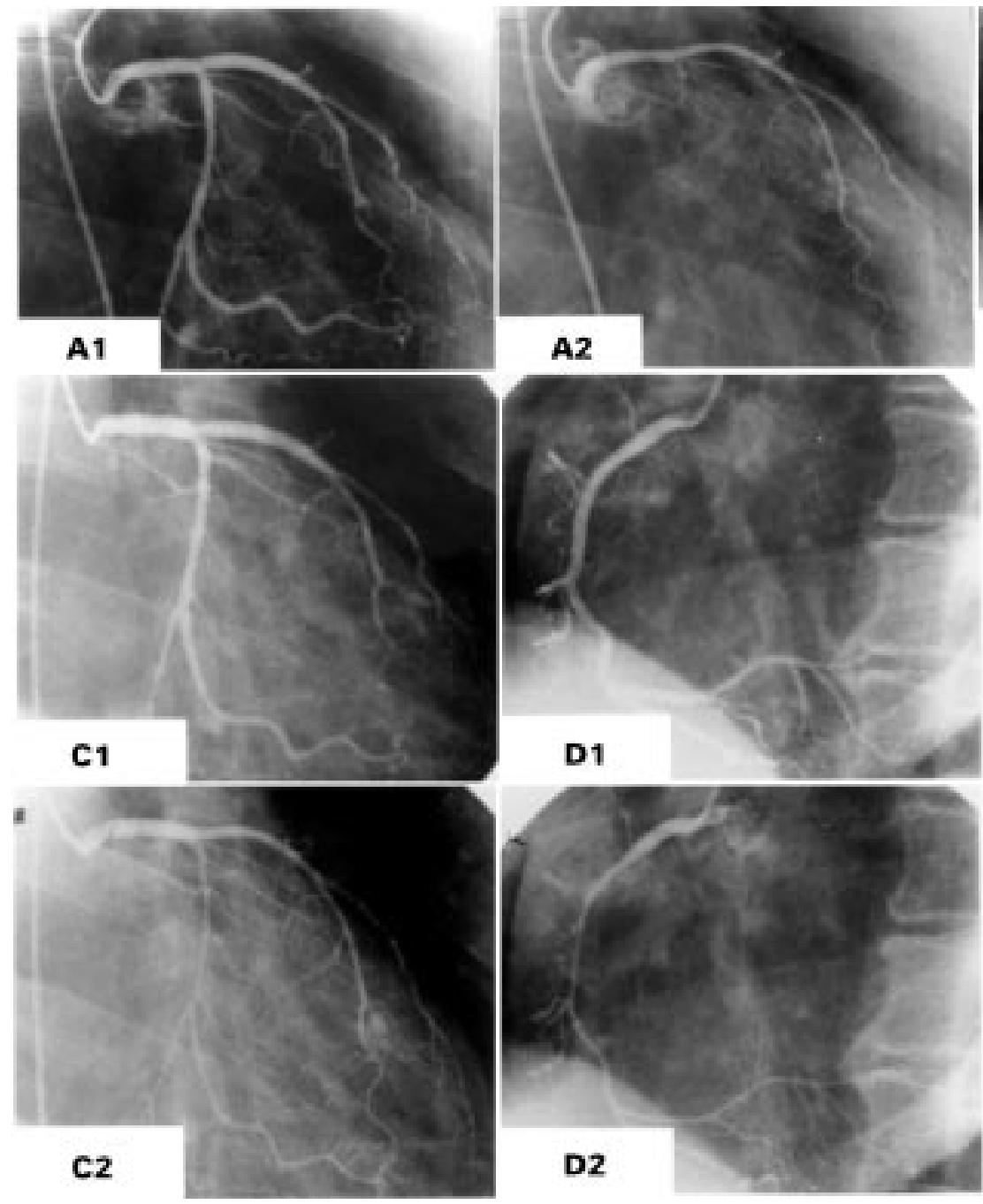

myocardial infarction were considered to be well controlled by our drug treatment regimen, which included sustained release isosorbide dinitrate given for more than two years. Of these 37 patients with pure vasospastic angina, 29 agreed to follow up coronary angiography and underwent ergonovine provocation during procedure. Two patients were excluded be-
Initial test

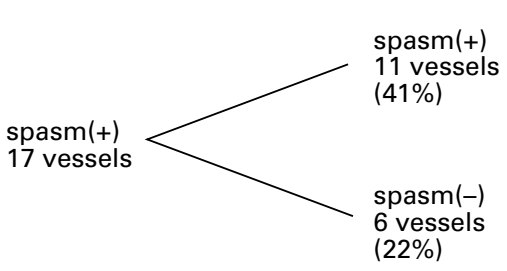

27 vessels

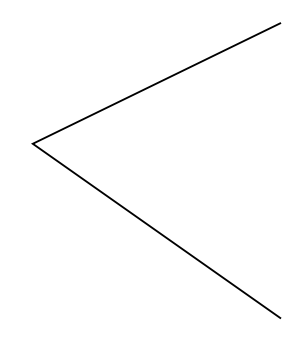

Follow up test

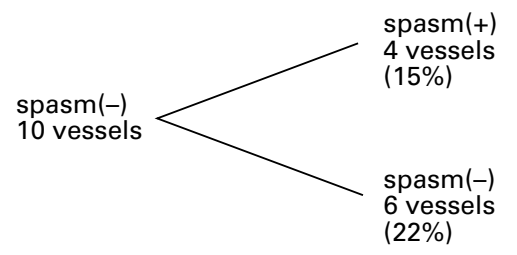

Figure 3 Results of ergonovine provocation tests in the left coronary artery.

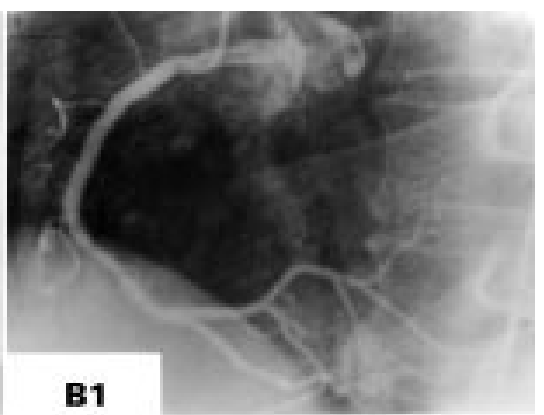

Figure 2 Initial and follow up coronary angiograms from patient 23. Angiograms of $A 1, A 2, C 1$, and $C 2$ of the left coronary artery were obtained in the right anterior oblique projection; angiograms B1, D1, and $D 2$ of the right coronary artery were obtained in the left anterior oblique projection. Angiograms were obtained after administration of $20 \mu \mathrm{g}$ of intracoronary ergonovine (A2, $C 2$, and D2), or after administration of $5 \mathrm{mg}$ of intracoronary isosorbide dinitrate $(A 1, B 1, C 1$, and $D 1)$. On the initial angiogram, occlusive spasm was observed in American Heart Association segment 13 after the administration of ergonovine (A2), but no significant organic stenosis was present after administration of isosorbide dinitrate (A1 and B1). On the follow up angiogram, spasms observed after administration of ergonovine were not

occlusive but caused $75 \%$ diffuse narrowing of the left and the right coronary arteries (C2 and D2).

cause their clinical records showed that they had had anginal attacks during the previous 24 months. Details of the other 27 patients are given in table 1 . There were 25 men and two women, mean (SD) age 63.4 (9.6) years at follow up coronary angiography.

\section{STUDY DESIGN}

All patients gave informed consent to the study. Both the first and the second ergonovine provocation were performed using the same uniform protocol reported previously. ${ }^{9}$ All coronary vasodilating drugs (nitrates, calcium blockers, and so on) were discontinued more than 48 hours before the examination, except for sublingual glyceryl trinitrate as needed. Coronary angiography was performed by the Judkins technique using $4 \mathrm{~F}$ or $5 \mathrm{~F}$ catheters. Ergonovine maleate solution, $0.2 \mathrm{mg} / \mathrm{ml}$, was diluted in $9 \mathrm{ml}$ of saline, and $1 \mathrm{ml}$ of this solution (20 $\mu \mathrm{g}$ ergonovine) was then given into each coronary artery by rapid bolus injection. This provocation test was carried out using the following procedure, under continuous monitoring of arterial blood pressure and ECG:

- Control angiography of the right coronary artery

- Control angiography of the left coronary artery 
- Angiography of the left coronary artery four minutes after the $20 \mu \mathrm{g}$ ergonovine dose had been given

- Angiography of the right coronary artery four minutes after the $20 \mu \mathrm{g}$ ergonovine dose had been given

- Angiography of the right coronary artery and the left coronary artery two minutes after administration of isosorbide dinitrate (5 mg).

Whenever chest pain or significant ST segment changes were observed, a selective coronary angiogram was immediately obtained. When occlusive spasm was observed, isosorbide dinitrate was given into the coronary artery and further provocation was withheld. In our ergonovine provocation test protocol, the left coronary artery was provoked under the same controlled conditions in the initial and follow up tests. To investigate any changes in spasticity, the incidence of left coronary artery spasm was compared between the initial test and the follow up test.

\section{Results}

The mean (SD) follow up period was 47.2 (21.6) months. Throughout the follow up period, 15 patients had been free from angina attacks. Four patients (cases 11, 17, 18, and 22) had atypical chest symptoms but these were considered not to be anginal on account of their characteristics and the lack of effect of glyceryl trinitrate. Three patients (cases 9, 19, and 23) had anginal attacks early during the follow up but had been free from attack for more than 24 months. Five patients (cases 3, $11,13,16$, and 27) experienced an anginal attack on accidentally omitting their drugs on one or more occasions. No patient was admitted to hospital as an emergency because of angina during the follow up period.

Seven patients (cases $2,3,4,6,15,21$, and 22) underwent ambulatory ECG monitoring during the follow up, and no ST segment changes were documented. Although two patients (cases 3 and 14) had suffered from headache as a side effect of isosorbide dinitrate, case 3 was able to keep taking the drug, while case 14 was able to tolerate it when given transdermally rather than orally.

After the follow up period, there was only one case (case 27) in whom organic stenosis developed. Seven patients (cases 2, 7, 12, 15, 18 , and 25) had an anginal attack during the wash out period of 48 hours before coronary angiography. Each attack was relieved easily by sublingual glyceryl trinitrate. Occlusive spasm was induced in 23 patients, at an earlier stage than in the initial test in four patients, unchanged in 10, and later in the other three patients (for example, fig 1). However, occlusive spasm was not induced in four patients; spasm as $90 \%$ narrowing was observed in three of these (cases 6, 9, and 14), and diffuse significant narrowing was observed in the other (case 23, fig 2). Occlusive spasm was induced in 17 of 27 left coronary arteries in the initial test, and in 15 of 27 left coronary arteries in the follow up test (fig 3). No significant difference was found between the initial test and the follow up test in left coronary artery spasticity (McNemar test; $\mathrm{p}=0.75$ ).

\section{Discussion}

ERGONOVINE PROVOCATION AND PATIENT SELECTION

Heupler et al reported ergonovine provocation using the systemic administration of $0.2-0.3$ mg of ergonovine maleate. ${ }^{11}$ We reported that using a $16 \mu \mathrm{g}$ dosage into each coronary artery was as sensitive as, and safer than, a systemic dose of $0.4 \mathrm{mg}$. ${ }^{9}$ Since 1988 , we have been performing routine ergonovine provocation, using $20 \mu \mathrm{g}$ into each coronary artery, at the time of first coronary angiography in patients with angina. In the present study, we undertook repeated provocation during coronary angiography in well controlled patients. Because we used the same regimen, we could compare the results between the initial and the follow up tests; the earlier the spasm was induced, the greater the spasticity was considered to be. We excluded patients with organic stenosis, because we were attempting to answer the question of whether medication should be continued indefinitely in well controlled patients with vasospastic angina.

Whittle et al concluded that the ECG response alone was an inadequate indicator of the presence of coronary artery spasm ${ }^{12}$; thus we did not use non-invasive assessments (for example, ambulatory ECG monitoring) in our assessment of vasospastic angina. In any event, because the patients had been well controlled by drug treatment, no ST segment changes on ambulatory ECG monitoring were documented during the follow up period.

REMISSION OF VASOSPASTIC ANGINA

The use of slow release isosorbide dinitrate in Japan since 1981 has made it easy to prevent anginal attacks in patients with vasospastic angina. Most patients with vasospasm but without significant coronary artery stenosis can be managed by drug treatment. Some studies have shown spontaneous remission. ${ }^{413-16}$ Waters et al reported that in many patients with vasospastic angina, the symptoms would disappear spontaneously, so drugs could safely be discontinued in some patients. ${ }^{8}$ Ergonovine testing would be useful in identifying those patients who could discontinue medication. The patients enrolled in our study were free from anginal attacks with the drug treatment regimen used. Spasm of either the left or the right coronary artery was induced by ergonovine provocation in most of these patients at follow up angiography. No significant difference was found between the initial and the follow up tests in the degree of spasticity of the left coronary artery. We diagnosed vasospastic angina only when spasm was confirmed on angiography, whereas Waters et al made the diagnosis using symptoms and ECG changes. This difference in diagnostic technique may have been responsible for the discrepant findings in the two studies. Although a positive ergonovine response in asymptomatic patients does not necessarily mean that the disease is 
sufficiently active to cause symptoms, we postulate that non-inducibility of spasm is likely to indicate remission of vasospastic angina.

Ozaki et al reported fluctuation in the location of the spasm in the coronary arteries. They concluded that, while vasospastic angina may be a transient disease, it may also be a persistent and variable condition lasting for many years. ${ }^{17}$ Our data suggest that anginal attacks may well recur after withdrawal of drugs, even in patients who have been symptom-free on medical management for several years. We feel that this risk should be emphasised if the question of discontinuing drug treatments is raised in patients with coronary vasospasm.

PROGRESSION OF STENOSIS

A correlation between coronary artery spasm and the initiation or progression of organic stenosis has been reported. ${ }^{18-23}$ However, in our series of 27 patients, coronary artery stenosis was discovered in only one case. Ozaki et al reported progression of stenosis in 16 patients who had been symptomatic during the follow up period. ${ }^{22}$ Also they reported regression of stenosis in 14 patients who had been free from anginal attacks and in whom spasm was not induced during follow up coronary angiography. Our patients were selected on the basis that they were well controlled by drug treatment, though spasm could be induced at follow up coronary angiography in most of them. Stenosis may not progress in patients who are well controlled, even if spasticity persists. Although on average the patients in our study were followed up for a long period, four $(15 \%)$ of the 27 patients had a follow up period of only 24 months. This may have implications with respect to disease progression.

REBOUND PHENOMENON

A rebound phenomenon caused by abrupt cessation of vasodilator drug treatment may influence the likelihood of spasticity at follow up coronary angiography. It was difficult to allow a longer cessation period to establish that there was no rebound, because we would have needed to admit the patients to hospital as a precaution throughout the cessation period, owing to the risk of an ischaemic attack. Thus a rebound phenomenon cannot be completely excluded.

CONCLUSIONS

Repeated ergonovine provocation and coronary angiography during a symptom-free period of several years' duration on drug treatment showed that coronary artery spasticity persisted in most cases. Though such patients may be free from symptoms, discontinuing their drug treatment may put them at high risk of ischaemic attacks.

1 Maseri A, L'Abbate A, Baroldi G, et al. Coronary vasospasm as a possible cause of myocardial infarction. $N$ Engl $\mathcal{F} M e d$ 1978;299:1271-7.

2 Oliva BP, Breckinridge JC. Arteriographic evidence of coronary arterial spasm in acute myocardial infarction. Circulation 1977;56:366-74.

3 Cipriano PR, Koch FH, Rosenthal SJ, et al. Clinical course of patients following the demonstration of coronary artery spasm by angiography. Am Heart f 1981;101:127-34.

4 Silverman CB, Heupler FA. Natural history of pure coronary artery spasm in patients treated medically. $\mathcal{F} \mathrm{Am} \mathrm{Coll}$ Cardiol 1983;2:200-5.

5 Nakamura M, Takeshita A, Nose Y. Clinical characteristics associated with myocardial infarction, arrhythmias, and sudden death in patients with vasospastic angina. Circulation 1987;75:1110-16.

6 Schroll JM, Veau P, Benacerraf A, et al. Long-term prognosis of medically treated patients with vasospastic angina and no fixed significant coronary atherosclerosis. Am Heart f 1988;115:559-64.

7 Yasue H, Takizawa A, Nagao M, et al. Long-term prognosis for patients with variant angina and influential factors. Circulation $1988 ; 78: 1-9$.

culation 1988;78:1-9.
8 Waters DD, Szlachcic J, Theroux P, et al. Ergonovine testing to detect spontaneous remissions of variant angina during long-term treatment with calcium antagonist drugs. $A m \mathcal{F}$ Cardiol 1981;47:179-84

9 Ueda O, Okazaki H, Kohchi K, et al. Intracoronary administration of ergonovine maleate for detecting vasospastic angina; one dose method. [Japanese with English abstract.] Kokyu to Funkan 1991;39:673-7.

10 American Heart Association Committee Report. A reporting system on patients evaluated for coronary artery disease. Circulation 1975;51:7-34.

11 Heupler FA, Proudfit WL, Razavi M, et al. Ergonovine maleate provocative test for coronary arterial spasm. $A m \mathcal{F}$ Cardiol 1978;41:631-40.

12 Whittle JL, Feldman RL, Pepine CJ, et al. Variability of electrocardiographic responses to repeated ergonovine provocrocardiographic responses to repeated ergonovine provocation in variant angina patients with

13 Schick EC, Liang C, Heupler FA, et al. Randomized withdrawal from nifedipine: placebo-controlled study in patients with coronary artery spasm. Am Heart f 1982;104: 690-7

14 Waters DD, Bouchard A, Theroux P. Spontaneous remision is a frequent outcome of variant angina. $7 \mathrm{Am}$ Coll Cardiol 1983;2:195-9.

15 Freedman SB, Richmond DR, Kelly DT. Long-term follow-up of verapamil and nitrate treatment for coronary artery spasm. Am f Cardiol 1982;50:711-15.

16 Kannel WB, Sorlie PD. Remission of clinical angina pectoris: the Framingham study. Am f Cardiol 1978;42: $119-23$.

17 Ozaki Y, Keane D, Serruys PW. Fluctuation of spastic location in patients with vasospastic angina: a quantitative angiographic study. $\mathcal{F}$ Am Coll Cardiol 1995;26:1606-14.

18 Marzilli M, Goldstein S, Trivella MG, et al. Some clinical considerations regarding the relation of coronary vasospasm to coronary atherosclerosis: a hypothetical pathogenesis. Am f Cardiol 1980;45:882-6.

19 MacAlpin RN. Relation of coronary arterial spasm to sites of organic stenosis. Am f Cardiol 1980;46:143-53.

20 Nobuyoshi M, Tanaka M, Nosaka H, et al. Progression of coronary atherosclerosis: is coronary spasm related to progression? f Am Coll Cardiol 1991;18:904-10.

21 Yamagishi M, Miyatake K, Tamai J, et al. Intravascular ultrasound detection of atherosclerosis at the site of focal vasospasm in angiographically normal or minimally narrowed coronary segments. F Am Coll Cardiol 1994;23: narrowed.

22 Ozaki Y, Keane D, Serruys PW. Progression and regression of coronary stenosis in the long-term follow-up of of coronary stenosis in the long-term follow-

23 Kaski JC, Tousoulis D, McFadden E, et al. Variant angina pectoris role of coronary spasm in the development of fixed coronary obstructions. Circulation 1992;85:619-26. 